\title{
Criminologie
}

\section{Examen critique des influences environnementales et familiales de la délinquance}

\section{David P. Farrington}

Volume 27, numéro 1, 1994

Analyse spatiale du crime

URI : https://id.erudit.org/iderudit/017346ar

DOI : https://doi.org/10.7202/017346ar

Aller au sommaire du numéro

Éditeur(s)

Les Presses de l'Université de Montréal

ISSN

0316-0041 (imprimé)

1492-1367 (numérique)

Découvrir la revue

Citer cet article

Farrington, D. P. (1994). Examen critique des influences environnementales et familiales de la délinquance. Criminologie, 27(1), 23-48.

https://doi.org/10.7202/017346ar
Résumé de l'article

The focus in this paper is on individual factors such as high impulsivity and low intelligence, family factors such as poor parental supervision and erratic parental discipline, and neighbourhood factors such as physical deterioration and social disorganisation. In the interest of reducing complexity, biological, peer, school and other factors will be neglected. 
The focus in this paper is on individual factors such as high impulsivity and low intelligence, family factors such as poor parental supervision and erratic parental discipline, and neighbourhood factors such as physical deterioration and social disorganisation. In the interest of reducing complexity, biological, peer, school and other factors will be neglected.

Le devenir délinquant peut être influencé par diverses catégories de facteurs : biologiques, individuels, familiaux, scolaires, environnementaux, démographiques, relatifs aux pairs, etc. Lorsqu'on se penche sur ce qui influence le développement de la délinquance, on est confronté au fait que la plupart des facteurs criminogènes identifiés sont interreliés. Par exemple, les adolescents issus de milieux physiquement détériorés et socialement désorganisés ont aussi tendance à provenir de familles qui exercent peu de surveillance sur leurs enfants: ils tendent également à être impulsifs et moins intelligents que la moyenne (cf. West et Farrington, 1973). La coexistence de ces types de problèmes complique la démonstration que leur rôle dans la délinquance est soit indépendant, soit interactif, soit séquentiel. Nous tenterons de démontrer que si aucune recherche n'a réussi à conclure sur cette question, $c$ 'est qu'aucune d'entre elles n'a mesuré simultanément les principaux facteurs individuels, familiaux et environnementaux. Comme le soulignent Tonry et al. (1991, p. 42) :

Les recherches passées se sont penchées sur les influences qui s'exercent ou bien sur les individus, ou bien sur les communautés, presque jamais sur les deux... Par conséquent, la plupart des recherches qui portent sur les individus sont inadéquates parce qu'elles négligent les variations dans les caractéristiques communautaires, tandis que celles qui se siment au plan communautaire négligent de tenir compte des différences individuelles.

Je m'attarderai particulièrement aux influences qui s'exercent sur la délinquance individuelle plutôt que sur les taux de criminalité ou de victimisation des collectivités. Je me concentrerai également sur les types d'infractions qui conduisent le plus fréquemment à l' arrestation et à la mise en accusation - et qui préoccupent le public - soit les vols simples,

1. Traduit de l'anglais par Suzanne Laflamme-Cusson.

2. Professeur, Institute of criminology, Cambridge University, 7 West road, Cambridge CB3 9DT, Angleterre. 
cambriolages, vols qualifiés, la violence, le vandalisme, l'abus de drogue et les fraudes mineures, délaissant la criminalité «en col blanc», faute de données de recherche pertinentes. Je m'intéresse à la délinquance juvénile et adulte mais ma préoccupation pour les influences familiales privilégie l'étude de la délinquance juvénile.

On peut dire que la délinquance surgit de l'interaction d'un individu ayant des tendances antisociales ou un potentiel criminel et d'un milieu qui présente des occasions criminelles. Les influences sur la propension au crime s'exercent à long terme et d'un individu sur l'autre, tandis que les facteurs du passage à l'acte jouent généralement de manière conjoncturelle, à court terme, et sur l'individu. Les variables qui agissent sur la propension au crime le font généralement en stimulant, en orientant ou en inhibant le comportement. De son côté, le passage à l'acte suppose un processus de prise de décision qui tienne compte des conséquences, des coûts et des bénéfices (cf. Farrington, 1992b, 1993). Pour rendre les choses moins complexes, je vais m'attarder aux influences individuelles, familiales et environnementales à long terme et sur le potentiel criminel, négligeant les influences conjoncturelles sur le passage à l'acte.

Quels sont les principaux facteurs individuels, familiaux et environnementaux? Diverses variables appartenant à chacune de ces catégories peuvent avoir divers effets. Par exemple certains facteurs environnementaux peuvent avoir des effets directs sur la délinquance tandis que d'autres peuvent avoir des effets indirects, par l'intermédiaire de facteurs familiaux. Certaines variables (pauvreté, chômage, classe sociale ou ethnie) peuvent être mesurées simultanément chez l'individu, dans la famille et dans l'environnement et peuvent avoir différents effets selon la catégorie. Cependant, les intercorrélations élevées des variables individuelles, familiales et environnementales les rendent difficiles à démêler. Par exemple, les individus de classe défavorisée ont tendance à vivre dans des familles pauvres et dans des quartiers pauvres. Il est important d'étudier l'impact des variables individuelles, familiales et environnementales sur l'encouragement, l'orientation et l'inhibition de la délinquance et sur diverses caractéristiques de la carrière criminelle comme son émergence, sa persistance et son abandon (Farrington, 1992a).

La plupart des théories proposent une chaîne séquentielle dans laquelle les facteurs environnementaux influencent indirectement plutôt que directement la délinquance. Par exemple, Trasler (1962) a suggéré que le principal facteur d'inhibition de la délinquance était l'anxiété conditionnée, laquelle dépend des méthodes d'éducation, et que ces dernières diffèrent dans les familles de classe moyenne et de classe défavorisée, ou selon les milieux (p. 77-81). Elliot et al. (1985-1989) ont suggéré que l'écart entre les aspirations et les possibilités réelles, une socialisation déficiente (dans les 
familles) et la désorganisation sociale (dans le milieu) affaiblissent les liens qui poussent à la conformité, ce qui renforce les liens délinquants et, en dernier lieu, la conduite délinquante. Reiss (1986) deffend l'idée que les quartiers de taudis favorisent une concentration de ménages dirigés par une femme seule, lesquels engendrent la faiblesse des contrôles parentaux, qui à son tour fait place à l'influence des pairs et à la sous-culture délinquante et finalement à la délinquance.

Nous commencerons par rappeler la recherche de Shaw et McKay (1942) souvent citée pour avoir démontré le rôle de l'environnement dans la délinquance ainsi que d'autres recherches plus récentes dans la même tradition. Puis nous regarderons la recherche de Glueck et Glueck (1950) qu'on peut voir comme une démonstration des influences individuelles et familiales sur la délinquance, ainsi que d'autres recherches dans le même esprit. Nous discuterons ensuite les questions plus générales de la théorie et de la mesure des facteurs individuels, familiaux et environnementaux et j'en tirerai quelques implications pour la prévention et le contrôle de la délinquance. Enfin, je ferai quelques suggestions de recherches dont on a besoin pour démontrer de manière plus concluante ces trois types d'influences.

\section{LA RECHERCHE DE SHAW ET McKAY}

Depuis Delinquency Areas (Shaw et al., 1929) jusqu'à Juvenile Delinquency and Urban Areas (Shaw et McKay, 1942, 1969), Shaw et McKay ont tenté d'expliquer les corrélations observées entre, d'une part, les taux de délinquance juvénile de différents quartiers de Chicago (et d'autres villes) et, d'autre part, des variables tirées des recensements (pauvreté des habitations, densité de population, occupation du propriétaire, composition ethnique) ainsi que les taux de problèmes socio-sanitaires comme l'absentéisme scolaire, les troubles mentaux, la tuberculose et la mortalité infantile. Les taux de délinquance juvénile considérés étaient ceux du lieu de résidence des délinquants et non du lieu des infractions - plus précisément les tau $\mathrm{x}$ du secteur de recensement auquel correspondait l'adresse des jeunes comparaissant devant le tribunal de la jeunesse.

Shaw et McKay ont conclu que les taux de délinquance étaient plus élevés au centre-ville et diminuaient à mesure qu'on s'éloignait vers la banlieue. Généralement, les quartiers à délinquance élevée avaient tendance à avoir aussi des taux élevés de problèmes socio-sanitaires et à être physiquement détériorés et socialement désorganisés. Un de leurs résultats qui a beaucoup moins attiré l'attention (sauf celle de Wilkström, 1991, p. 141) est qu'une grande proportion de tous les délinquants proviennent d'une petite fraction de quartiers. Ce résultat nous rappelle d'une certaine manière 
une conclusion de West et Farrington (1977) à l'effet qu'environ $4 \%$ des familles étaient impliquées dans la moitié des accusations et une conclusion de Sherman et al. (1989) à l'effet que la moitié des appels à la police provenaient de $3 \%$ des adresses. La disproportion est souvent moindre dans le cas des quartiers : par exemple, Hamparian et al. (1978) ont trouvé qu'un tiers de leur cohorte de jeunes violents de Columbus, Ohio, vivait dans 10 secteurs de recensement (à prédominance pauvre et noire) représentant $8 \%$ de la population.

Comme Short (1969) l'a noté dans son introduction à l'édition révisée de Juvenile Delinquency and Urban Areas, la conclusion la plus controversée de Shaw et McKay est à l'effet que la concentration de la délinquance au centre-ville a persisté sur une longue période (des années 1900 aux années 1960) malgré des vagues d'immigration successives de gens d'origine et d'ethnies différentes dans ces quartiers. En 1900, les résidents du centre-ville étaient principalement d'origine allemande, irlandaise ou scandinave. Puis ces groupes se sont déplacés et ont été remplacés par des immigrants d'Europe de l'est (en particulier des Polonais); puis les Italiens ont succédé aux Polonais et ils furent à leur tour remplacés par les Noirs du sud. Devant la constance dans le temps des taux de délinquance des divers quartiers, Shaw et McKay ont prétendu que la délinquance était d'abord influencée par les caractéristiques physiques et sociales, indépendamment du type d'individus et de familles vivant dans les quartiers.

Shaw et McKay ont conclu (1942, p. 435) que :

Le fait que les taux de délinquance à Chicago soient demeurés relativement constants dans les secteurs adjacents au commerce et à l'industrie, malgré des changements successifs de population en termes d'ethnie et de nationalité, mène nécessairement à la conclusion que les facteurs de délinquance sont inhérents à la communauté.

On ne saurait trop insister sur l'importance de cette conclusion dans l'histoire de la criminologie. C'est éminemment à cause d'elle que la criminologie, qui jusqu'aux années 20 était dominée par les psychologues et ìes psychiatres, est passée sous la domination des sociologues dans les années 30. Le sociologue le plus influent dans ce mouvement fut Sutherland, auteur du non moins influent manuel de criminologie (Principes de Criminologie) entre 1930 et 1970 et, peut-être le plus important sociologue du crime au vingtième siècle.

Ce qu'on lit dans le manuel de Sutherland (1939, p. 138-142) à propos de la recherche de Shaw et McKay est fort éclairant :

...dans les districts où la criminalité était élevée en 1930, elle l'était également en 1900 malgré des bouleversements souvent radicaux survenus dans l'origine nationale de la population du district au cours de cette période... 
On a donné deux explications principales de la concentration des délinquants. La première est fondée sur la structure sociale du voisinage. Les zones de concentration de la délinquance dans les grandes villes américaines, et surtout à Chicago où la question a été étudiée avec le plus d'attention, sont les zones malsaines, surpeuplées, habitées par une population en régression. économiquement faible et composée en grande partie de Noirs et d'étrangers qui ne sont que locataires de leur logement, et où il existe peu de services sociaux financés par les résidents locaux. L'infraction aux lois y est devenue traditionnelle... La seconde interprétation a été soutenue par les psychiatres. Les zones dans lesquelles le taux de la délinquance est élevé sont celles où les loyers sont bas; la raison en serait que la partie de la population qui est ainsi définie par le montant des loyers qu'elle peut payer, comporte une part importante de psychopathes. La concentration des délinquants est alors interprétée comme due à une ségrégation ou à une migration sélective... Contre l'interprétation qui attribue les zones de délinquance à la ségrégation. l'argument le plus frappant est la découverte par Shaw de la stabilité presque parfaite du taux de la délinquance au cours d'une période de trente années, malgré un bouleversement presque complet de la composition de la population. ce qui indique que le taux de la délinquance est fonction de la zone d'habitation plutôt que du type de ses habitants ${ }^{3}$.

Selon Shaw et McKay $(1942,1969)$, donc, la délinquance dépend de caractéristiques de l'environnement et non de caractéristiques individuelles. Ils donnent plusieurs explications sur le comment et le pourquoi de l'influence du milieu sur la délinquance, dont la plus connue probablement concerne le lien entre la détérioration physique et la «désorganisation sociale»: la faible capacité qu'ont les institutions locales de contrôler le comportement des résidents permet l'émergence de la délinquance. Le contrôle communautaire consiste par exemple à interroger les gens sur leurs activités suspectes ou à réprimander les enfants pour leurs comportements inacceptables (Cf. Bursik, 1988 pour un examen du concept de désorganisation sociale). Cependant Shaw et McKay ont aussi suggéré que, dans les quartiers délinquants, les traditions de délinquance étaient transmises d'une génération à l'autre à travers les associations différentielles (p. 174), que les familles étaient incompétentes (p. 183), qu'il y avait un écart entre les visées et les possibilités réelles sur le plan économique (p. 186), et qu'il existait des gangs et des sous-cultures. De manière plus générale, les auteurs mettaient l'accent sur la transmission culturelle des valeurs antisociales et sur l'inefficacité du processus de socialisation, dans les milieux désorganisés.

3. Traduction de Principes de criminologie aux éditions Cujas, Paris, 1966. 


\section{Le statut actuel de la recherche de Shaw et Mckay}

La recherche de Shaw et McKay est encore souvent citée dans la criminologie américaine (par exemple Cohn et Farrington, 1990). Néanmoins, elle a été l'objet de plusieurs critiques, notamment sur le problème évident de la validité des dossiers de la cour juvénile comme mesure de la délinquance et sur l'arbitraire de la mesure d'un mille carré, à Chicago, comme critère d'homogénéité du milieu. La plupart des critiques figurent dans le bilan réalisé par Gold (1987) sur la contribution de Shaw et McKay.

Un problème majeur est ce qu'il est convenu d'appeler l'erreur écologique. Comme Robinson (1950) l'a souligné, on ne peut pas nécessairement conclure à l'existence de corrélations sur le plan individuel à partir de l'observation de "corrélations écologiques» (corrélations de taux relatifs à des zones). Robinson (1950, p. 352), faisant explicitement référence à Shaw et McKay, a énoncé que, «dans chaque étude où figurent des corrélations écologiques, on les utilise de toute évidence en vue de découvrir quelque chose sur le comportement des individus». Par exemple, Shaw et McKay défendent l'idée que «les variations locales dans la conduite des enfants, telles que révélées par les taux différentiels de délinquance, reflètent des différences dans les valeurs, les normes et les attitudes sociales auxquelles sont exposés les enfants 》. En d'autres termes, Shaw et McKay ont voulu tirer des conclusions relatives aux influences de l'environnement sur la délinquance individuelle, alors que leur utilisation de corrélations écologiques ne les y autorisait pas toujours.

Un autre problème : Wallis et Maliphant (1967) ont trouvé que les taux de délinquance des quartiers de Londres étaient négativement corrélés aux taux de divorce de ces quartiers, cependant que les sujets délinquants de leur étude avaient plus souvent des parents divorcés qu'un échantillon représentatif de garçons. Cela peut être dû en partie au fait que des taux se rapportant aux secteurs de recensement peuvent refléter des comportements des gens âgés de ces secteurs tandis que les taux de délinquance reflètent des comportements des gens plus jeunes. Curieusement, Glaser et Rice (1959) ont démontré que les taux de chômage étaient à la fois positivement corrélés aux taux de criminalité adulte et négativement corrélés aux taux de délinquance juvénile. Firebaugh (1978) a passé en revue les conditions dans lesquelles on pouvait - ou on ne pouvait pas - tirer des conclusions valides au plan individuel à partir de corrélations observées à des niveaux agrégés.

Pour estimer les influences du milieu sur la délinquance, je mettrai l'accent sur le fait de la constance relative des taux de délinquance dans différents milieux, sans égard pour les types d'habitants. C'est à Jonassen (1949) qu'on doit la critique la plus dévastatrice de la conclusion de Shaw et McKay sur le peu d'importance de l'origine ethnique et nationale. Il a 
souligné qu'il fallait non seulement comparer les taux de délinquance des mêmes groupes ethniques dans différents milieux (comme Shaw et McKay) mais aussi de divers groupes ethniques dans le même environnement. Jonassen soutient que les données de Shaw et McKay indiquent que, dans les mêmes quartiers de Chicago, les groupes originaires du Nord et de l'Ouest européen ont des taux de délinquance plus bas que les groupes originaires du Sud et de l'Est de l'Europe. Jonassen a aussi noté que Shaw et McKay (1942, p. 440) avaient également trouvé que les Orientaux avaient des taux relativement bas, même dans les zones très détériorées. tandis que les Noirs, où qu'ils vivent, avaient des taux de délinquance relativement élevés. Jonassen $(1949$, p. 613$)$ affirme :

Des contradictions aussi évidentes sont difficiles à comprendre, sauf à soupçonner l'existence de biais cachés qui conduisent à la distorsion des données dans certaines directions. On sent la trame du déterminisme 6́cologique... On sent également dans la trame de leur raisonnement des poussées idéologiques qui les retiennent, même lorsqu'ils sont tentés par les données empiriques, de pécher contre «l'idéologie professionnelle des pathologistes sociaux » ou contre la démocratie, en invoquant la possibilité que tous les groupes ethniques, raciaux ou nationaux ne soient pas également aptes à résister à la «désorganisation » qu'est la délinquance juvénile.

Shaw et McKay (1949, p. 617) ont rétorqué :

On nous a accusés d'avoir laissé paraître nos préférences idéologiques et notre penchant démocratique dans nos études... Il est peut-être vrai... que nous ayons ressenti quelque satisfaction devant le fait que nos données suggèrent avec constance que, dans des circonstances comparables, les taux de délinquance des groupes mal cotés ne seraient pas différents des taux des groupes bien cotés... Le fait important est que les taux de délinquance des garçons noirs varient, eux aussi. selon le milieu. Ils sont plus élevés que ceux des garçons blancs mais on ne peut pas dire qu'ils sont superrieurs à ceux des garçons blancs de milieux comparables, parce qu'il est impossible de reproduire dans les communautés blanches les conditions de vie dans lesquelles vivent les Noirs.

Après avoir rapporté cet échange entre Jonassen et Shaw et McKay, Gold (1987, p. 77) conclut :

La contestation de Jonassen est valable. Shaw et McKay ont montré que les conditions sociales sont reliées aux taux de délinquance. sans égard aux groupes ethniques. Mais ils n'ont pas démontré que les conditions sociales expliquent toute la différence et l'ethnie, rien. Si les corrélations entre la composition ethnique et les taux s'affaiblissent lorsque les conditions économiques changent, elles ne disparaissent pas. Les groupes ethniques semblent traîner avec eux différents degrés de propension à la délinquance, 
au moins sur plusieurs générations, si jamais elles disparaissent. Cela peut être dû aux traits les plus persistants de leur culture, à des facteurs génétiques ou, en particulier chez les Noirs, à des conditions de vie inéluctablement mauvaises. Les données de Shaw et McKay ne permettent pas de conclure là-dessus.

Les recherches plus récentes sèment le doute sur la relative constance des taux de délinquance dans le temps. Dans son introduction à Juvenile Delinquency and Urban Areas, Short (1969) a noté que les quartiers dont la population blanche avait été complètement remplacée par une population noire à la fin des années 40 et dans les années 50 . connaissaient les plus grandes hausses des taux de délinquance (tandis qu'on observait les plus grandes décroissances dans les quartiers à prédominance noire depuis longtemps). Or Jonassen avait constaté depuis longtemps les mêmes tendances à la hausse.

...l'énude des données révèle que dans certains secteurs on trouve de fortes corrélations négatives plutôt que de fortes corrélations positives. Par exemple, lorsqu'on compare la carte no 9 qui indique le lieu de résidence des délinquants de 1934-1940 à la carte no 12 qui indique la même réalité en 1900-1906, il y a des différences marquées d'une part entre la $63^{\mathrm{e}}$ et la $31^{\mathrm{e}}$ rue, d'autre part entre Cottage Grove et State. On voit tout de suite qu'ici, on aurait un coefficient de corrélation négatif élevé - au-dessus de 90. Et ce changement surprenant est étroitement corrélé avec l'arrivée des Noirs dans ce secteur.

En somme, la recherche de Shaw et McKay ne démontre pas que les facteurs environnementaux jouent sur la délinquance indépendamment des facteurs individuels ou familiaux.

Parce que Shaw et McKay étaient des chercheurs méticuleux et que leurs données ont été conservées, Bursik et Webb (1982) ont pu tester la validité de leurs hypothèses en utilisant des méthodes quantitatives plus sophistiquées. Ils ont noté que Shaw et McKay avaient dû faire tous leurs calculs à la main et que leurs conclusions «étaient d'abord basées sur l'examen visuel des distributions sur les cartes, même s'ils avaient calculé quelques coefficients de corrélation» (1982, p. 28). Bursik et Webb ont conclu que les taux de délinquance relative n'étaient pas stables après 1950 et qu'ils reflétaient les changements démographiques. Les variations dans les taux de délinquance des divers quartiers étaient significativement corrélées aux variations des pourcentages des gens de couleur, des Blancs d'origine étrangère et des logements surpeuplés. Les plus fortes hausses dans les taux de délinquance se produisaient lorsque les Noirs passaient de minoritaires à majoritaires dans un secteur. D'où il semble que les taux de délinquance aient varié selon le genre d'individus vivant dans les divers secteurs 
de Chicago. Il serait intéressant de vérifier si on parviendrait aux mêmes résultats avec des mesures de délinquance et de comportement antisocial autres que les dossiers officiels.

Le nceud du problème de la recherche de Shaw et McKay est qu'on ignore dans quelle mesure les taux différentiels de délinquance des secteurs sont le reflet des variations des facteurs environnementaux comme la dégradation physique et la désorganisation sociale, et dans quelle mesure ils reflètent d'autres types de facteurs, individuels et familiaux, par exemple. On n'a jamais pu s'appuyer sur des bases solides pour rejeter l'hypothèse rivale, voulant que les taux de délinquance des quartiers soient tributaires d'une population à la dérive, migrante, dépendante (les familles pauvres, à problèmes multiples et antisociales se déplacent vers les secteurs à loyers modiques ou subventionnés). Par exemple, Wikström (1985), en Suède, au terme d'une recherche soignée utilisant une méthodologie sophistiquée, a conclu que les taux de criminalité de violence étaient élevés dans le centreville d'abord parce que ces quartiers attiraient des alcooliques, des drogués et des criminels reconnus).

Ni Shaw et McKay, ni aucun chercheur après eux n'a été capable de démontrer sans ambiguïté que les facteurs environnementaux exerçaient une influence importante sur la délinquance indépendamment des facteurs individuels ou familiaux. De même, aucun chercheur n'a étudié d'une manière satisfaisante les interactions des facteurs environnementaux et individuels susceptibles de jouer sur la délinquance, de manière à prouver ou à rejeter l'hypothèse d'une susceptibilité différentielle des groupes aux influences envirơnnementales.

\section{Les recherches plus récentes sur les influences environnementales}

On a réalisé un grand nombre de recherches inspirées de celle de Shaw et McKay sur les taux de délinquants et de délinquance dans divers milieux (cf. comptes rendus de Baldwin, 1979; Gold, 1987; Bursik, 1988). Beaucoup étaient de nature transversale, tandis que quelques-unes ont repris la méthode longitudinale de Shaw et McKay (Schmid, 1960; Baldwin et al., 1976 ; Bursik et Webb. 1982 ; Schuerman et Kobrin, 1986). Récemment, on a mis l'accent sur le comment et le pourquoi des changements à travers le temps des taux de délinquance des quartiers (les «carrières criminelles des communautés»: Reiss, 1986; Bottoms et Wiles, 1986) plutôt que sur la stabilité de ces taux.

Très peu de chercheurs s'inscrivant dans la tradition écologique de Shaw et McKay ont tenté d'étudier les influences individuelles, familiales et environnementales sur la délinquance individuelle. De telles études requièrent des données individuelles, alors que la plupart de ces chercheurs, à l'instar de Shaw et McKay. ont utilisé des données écologiques et ont mis 
l'accent sur les taux de délinquance (Sampson et Groves, 1989 ; Bursik et Grasmick, 1992). Les meilleures études à se situer à plusieurs niveaux sont probablement celles de Johnstone (1978), Simcha-Fagan et Schwartz (1986) et Gottfredson et al. (1991). On a eu également quelques études de ce genre sur la victimisation individuelle (Sampson et Lauritsen, 1990) mais je n'en parlerai pas ici. Pas plus que je ne parlerai des problèmes statistiques liés à l'usage de variables écologiques pour prédire des variables relatives aux individus.

Simcha-Fagan et Schwartz (1986) sont partis d'un modèle écologique. Constatant que les variables de recensement se regroupaient en deux ensembles - l'un indiquant la désorganisation familiale (familles monoparentales) et l'autre, l'opulence (le revenu du ménage) - ils ont étudié douze quartiers de New York, différents les uns des autres en termes de désorganisation sociale (élevée, moyenne et basse), de richesse (élevée ou faible), et à prédominance de Blancs ou de Noirs. Ils ont interviewé plus de 550 adolescents masculins et leurs mères puis recueilli des données écologiques et d'autres renseignements (particulièrement sur la fréquentation de pairs délinquants et l'attachement à l'école). Les variables écologiques qui se sont avérées les plus importantes dans leurs analyses n'appartenaient pas au modèle original mais avaient été tirées de leurs entrevues.

Par les analyses de régression, Simcha-Fagan et Schwartz ont trouvé que les variables indépendantes les plus fortement reliées à la délinquance officielle étaient l'âge, le revenu familial, et la désorganisation sociale dans la communauté (hautement corrélée à la situation monoparentale). Les variables indépendantes les plus fortement reliées à la délinquance autorévélée étaient l'âge, la stabilité résidentielle dans la communauté, et une faible participation aux organisations communautaires (apparemment le reflet du niveau d'éducation des parents). Cependant, il semble que d'autres variables importantes (pairs délinquants et attachement à l'école) aient été exclues de ces analyses, puisque Simcha-Fagan et Schwartz sont venus a la conclusion que les variables communautaires et familiales ont des effets indirects sur la délinguance, par l'intermédiaire de leurs effets sur les pairs délinquants et sur le détachement de l'école. Ils en infèrent que: "Les effets de la communauté sur la délinquance sont largement médiatisés par les expériences de socialisation » (1986, p. 695). Cependant, Simcha-Fagan et Schwartz ont tout simplement montré qu'un modele causal était consistant avec leurs données. Il n'est pas évident qu'ils aient mis à l'épreuve des modèles de causalité radicalement différents. De même, ils n'ont mesuré aucune des variables individuelles et familiales qui se sont révélées les plus importantes dans les études longitudinales comme l'impulsivité, l'intelligence, la supervision, la discipline et la criminalité des parents. 
Gottfredson et al. (1991) ont recueilli des données démographiques, familiales, écologiques, ainsi que des mesures de délinquance auto-révélée auprès de 3700 adolescents à l'école. Conformément aux résultats de Simcha-Fagan et Schwartz, les variables environnementales se sont regroupées en deux ensembles, l'un («désorganisation») reflétant les ménages à parent unique féminin, pauvre et assisté social et l'autre («la richesse») reflétant les emplois de professionnels ou de cadres ainsi que des niveaux élevés de revenus et l'éducation. Gottfredson et al. ont trouvé que, dans des analyses de régressions multiples où on incluait des variables environnementales parmi des variables démographiques, familiales, scolaires et relatives aux pairs, l'effet de l'environnement était très faible. Tandis que les corrélations entre les variables écologiques et la délinquance auto-révélée étaient de l'ordre de zéro, celles qui reliaient l'âge, l'existence de pairs déviants, l'attachement aux parents ou à l'école à la délinquance étaient bien plus élevées. Qui plus est, les relations n'étaient pas tout à fait telles que postulées, puisque le seul résultat significatif pour les garçons fut que ceux qui vivaient dans des milieux riches avouaient une délinquance plus élevée que les autres. Par contre, les garçons dont les parents avaient un niveau d'éducation plus élevé rapportaient moins de délinquance, ce qui a amené Gottfredson et al. à dire que la relation entre la classe sociale et la délinquance est inversée quand on passe du niveau environnemental au niveau individuel. Ils laissent également entendre en note infra-paginale qu'il y a interaction de ces deux niveaux de mesure de la classe sociale.

Le problème majeur de l'étude de Gottfredson et al. est qu'ils n'ont pas étudié les divers modèles possibles (e.g. séquentiel versus simultané) liant les variables démographiques, familiales. scolaires et relatives aux pairs aux variables écologiques. De plus, leurs mesures de la délinquance et des variables non environnementales provenaient toutes de données autorévélées, ce qui pouvait accentuer les relations à cause de biais communs. L'étude comportait également un éventail très limité de variables dont aucun trait individuel tel qu'une grande impulsivité ou une faible intelligence. Enfin, l'étude était transversale plutôt que longitudinale, de sorte que seules les variations inter-individuelles étaient saisissables.

Gottfredson et Taylor (1986) ont mené une étude intéressante à Baltimore sur l'effet sur la récidive, du contexte environnemental dans lequel un prisonnier était mis en liberté. Ils ont trouvé que les mesures écologiques (tirées de l'observation systématique) tombaient sous deux catégories : la dégradation du quartier (graffiti, déchets, logements inoccupés, groupes de jeunes flâneurs) et la vocation économique des zones (commerciale, industrielle ou institutionnelle). Une fois contrôlées les caractéristiques des délinquants (histoire criminelle et sociale), les facteurs environnementaux n'aidaient pas à prédire la récidive, ce que Reiss (1951a) avait trouvé plusieurs années auparavant. Cependant, il y avait des signes d'interaction 
entre les types de personnes et les types d'environnements, les pires délinquants (les plus criminalisés) se conduisant mieux lorsqu'ils recouvraient la liberté dans des milieux relativement bons et vice versa.

On peut difficilement s'empêcher de conclure que ta connaissance sur les influences individuelles, familiales et environnementales est plutôt fragmentaire.

\section{LA RECHERCHE DES GLUECK}

Au cas où on me croirait injustement critique à l'endroit des recherches sur les influences environnementales, $j$ 'aimerais préciser qu'on trouve des problèmes similaires dans les recherches sur les influences individuelles et familiales. Ces dernières ont négligé l'étude des facteurs environnementaux. Aucun projet n'a encore démontré de manière concluante que les facteurs individuels et familiaux influencent la délinquance indépendamment de (ou en interaction avec) les facteurs environnementaux. Il est concevable que des facteurs individuels et familiaux prédisent la délinquance seulement parce que leur prévalence varie dans les différents environnements et que les facteurs écologiques soient les plus importants. On peut aussi concevoir que les facteurs individuels et familiaux aient différents effets sur la délinquance selon les milieux.

Pour illustrer les problèmes de la recherche sur les facteurs individuels et familiaux, j'ai choisi de me reporter au travail des Glueck, pionniers de la recherche sur ce type de facteurs comme Shaw et McKay le furent relativement aux facteurs environnementaux. De toute évidence, les Glueck étaient des chercheurs encore plus mirutieux que Shaw et McKay (on en a conservé encore toutes les données brutes et de correspondance : Laub et Sampson, 1988, 1991) et ils sont encore plus cités dans la littérature criminologique récente (Cohn et Farrington, 1990). Il n'est probablement pas accidentel que le premier volume de la prestigieuse collection «Crime and Justice» qui produit des revues des écrits à la fine pointe des grandes questions criminologiques comprenne un chapitre sur les études environnementales (Baldwin, 1979), qui reconnaît la contribution de Shaw et McKay, et un autre sur les études longitudinales (Farrington, 1979) qui reconnaît la contribution des Glueck.

Les Glueck ont d'abord contribué au savoir criminologique à travers leurs recherches longitudinales sur les carrières criminelles, et particulièrement par leurs études suivies de 500 jeunes garçons dans les maisons de correction (Glueck et Glueck, 1930, 1937, 1943), 1000 jeunes délinquants de la cour (Glueck et Glueck, 1934, 1940), et 500 délinquants institutionnalisés jumelés avec 500 non délinquants (Glueck et Glueck 1968). Cependant ils sont probablement plus connus aujourd'hui pour leur recherche 
transversale Unraveling Juvenile Delinquency (Glueck et Glueck, 1950) et c'est sur elle que je mettrai l'accent. De plusieurs points de vue, il est dommage que ce soit leur étude la plus célèbre; non seulement elle est transversale mais elle a des prétentions exagérées sur la capacité de prédire la délinquance. Néanmoins ce livre contient des renseignements utiles sur à peu près tout ce qu'on peut concevoir comme facteurs de délinquance. Par exemple, lorsque je m'interrogeais récemment sur l'importance de l'âge de la mère à la naissance de son premier enfant en relation avec la délinquance de ses enfants, je me suis tourné vers Unraveling Juvenile Delinquency pour y trouver un tableau pertinent, p. 117.

Dans Unraveling Juvenile Delinquency, les Glueck ont tenté de jumeler cas par cas 500 délinquants institutionnalisés à 500 non-délinquants de Boston selon le sexe (tous des garçons), l'âge (à un an près), l'intelligence (10 points sur l'échelle Wechsler-Bellevue), l'origine ethnique et la résidence dans des quartiers pauvres (à $93 \%$ depuis leur naissance). Par leurs parents et grand-parents, ils étaient surtout d'origine britannique (26\% des paires), italienne $(25 \%)$, irlandaise $(19 \%)$ et américaine $(8 \%)$. Tous étaient blancs. Pour la sélection des non-délinquants, dans des écoles choisies, on écartait les petits délinquants persistants non appréhendés, de sorte que les groupes de délinquants et non-délinquants sont en quelque sorte des extrêmes. Dans ce pairage sur des variables environnementales, le but des Glueck était clairement de savoir si les facteurs individuels, familiaux et autres jouaient sur la délinquance indépendamment des caractéristiques environnementales.

Malheureusement, leur pairage de quartiers était moins bon que celui des autres facteurs, comme un jeune malin l'a fait remarquer à l'époque (Reiss, 1951 b). En effet, $70 \%$ des secteurs de recensement de Boston (109 sur 156) étaient classés défavorisés, la plupart du temps à cause de leur taux relativement élevé de délinquance juvénile. D'où pratiquement aucun délinquant institutionnalisé n'était soustrait de l'échantillon pour défaut d'appartenir à un quartier défavorise. Chaque non-délinquant devait provenir non pas du même secteur de recensement que son semblable délinquant mais seulement du même genre de zone défavorisée. Le pairage selon l'environnement se révềle inadéquat puisque $55 \%$ des délinquants (versus $34 \%$ des non-délinquants) vivaient dans des quartiers de taudis cependant que $49 \%$ des non-délinquants (versus $31 \%$ de délinquants) vivaient dans des zones mixtes (commerciales et résidentielles).

Les Glueck ont trouvé de nombreuses différences significatives entre les délinquants et les non-délinquants sur des facteurs individuels et familiaux (et autres). S'appuyant sur des méthodes statistiques pas tout à fait adéquates, ils ont conclu que les principaux prédicteurs de la délinquance, mutuellement exclusifs et mesurables dès l'âge de six ans étaient : une 
discipline paternelle erratique ou trop stricte, un manque de surveillance maternelle, des attitudes parentales indifférentes ou hostiles à l'endroit du garçon et la faiblesse de la cohésion familiale.

Sur la base d'entrevues psychiatriques, les principaux prédicteurs individuels mutuellement exclusifs se sont avérés le goût du risque, l'extraversion, la suggestibilité. l'entêtement et l'instabilité émotive. Néanmoins il demeure possible que les différences individuelles et familiales observées entre délinquants et non-délinquants ne soient qu'une conséquence des différences de milieu. En moyenne, les délinquants vivaient dans de plus mauvais quartiers.

\section{Le statut actuel de la recherche des Glueck}

Unraveling Juvenile Delinquency nous a fourni des renseignements de base sur un grand nombre de réalités liées à la délinquance, que toute théorie doit reconnaître et expliquer. Malgré tout, ce livre a fait l'objet de nombreuses critiques qu'on trouve pour la plupart dans Hirschi et Selvin (1967). À l'opposé de la recherche de Shaw et McKay dont les conclusions ont été acceptées pratiquement sans réserve par la criminologie américaine, celle des Glueck a été tant critiquée sur tant d'aspects qu'il n'est pas nécessaire que je m'y attarde dans le détail. Les plus grandes difficultés viennent de la nature transversale de l'étude qui soulève des problèmes si on veut parler de causalité. Par exemple comment savoir si les parents des délinquants sont devenus moins cohérents et plus hostiles aux garçons en réaction a leur délinquance persistante ou si ce sont la faible cohésion familiale et l'hostilité parentale qui sont d'abord apparues pour mener ensuite à la délinquance des garçons. Le biais rétrospectif est également un grand problème. Comment savoir si les différences mesurées entre délinquants et non-délinquants n'étaient pas influencées par la connaissance des interviewers de l'appartenance des sujets au groupe des délinquants ou des non-délinquants. Enfin les Glueck n'auraient pas dû tirer de conclusions sur la possibilité de faire des prédictions dès six ans, sur la foi de données recueillies en moyenne à 14 ans.

Ces critiques sévères de la recherche des Glueck ont inspiré une génération d'études prospectives longitudinales sur la délinquance, mettant l'accent sur les facteurs individuels et familiaux et visant à surmonter les difficultés évoquées, dont les plus importantes sont passées en revue par Farrington (1979, 1988a). Il est plutôt ironique qu'une recherche longitudinale cruciale et généralement admirée comme celle de Shaw et McKay (1942) ait inspiré une génération de répliques transversales et appauvries tandis que la recherche transversale tant critiquée des Glueck a, comme il se doit, inspiré une génération de recherches longitudinales de meilleure facture. Il est aussi intéressant de noter que la plupart des variables liées à la délinquance que les Glueck avaient cernées se soient avérées des 
prédicteurs de délinquance dans des études longitudinales de plus grande envergure. Gottfredson et Hirschi $(1987$, p. 582) ont écrit que «malgré une myriade de critiques sur le travail des Glueck, leurs trouvailles se sont avérées concordantes avec celles des recherches subséquentes " tandis que, selon Laub et Sampson (1991, p. 1143) les principales conclusions des Glueck sont correctes et Jes questions qu'ils ont posées restent des sujets majeurs en criminologie.

\section{La recherche récente sur le rôle des facteurs individuels et familiaux}

Farrington (1992b, 1992c) a passé en revue les principaux prédicteurs individuels, familiaux et autres de la délinquance (juvénile et adulte, officielle ou auto-révélée). Au plan individuel, ce sont une forte impulsivité (hyperactivité, agitation, manque de concentration, témérité), le manque d'intelligence et l'échec scolaire. Les principaux prédicteurs familiaux sont le manque de surveillance des parents, une discipline erratique ou excessive, des conflits entre les parents, la délinquance des parents ou de la fratrie ainsi que des perturbations familiales qui se manifestent par la séparation des enfants de leurs parents biologiques. Toute étude sur l'importance des facteurs individuels, familiaux et environnementaux, qu'on les considère indépendamment les uns des autres ou en interaction, doit inclure au moins ces dimensions.

Les auteurs de recherches longitudinales prospectives se sont habituellement plus intéressés aux facteurs individuels et familiaux qu'aux facteurs environnementaux et par conséquent n'ont pas accordé suffisamment d'attention aux influences écologiques. Comme le soulignent Simcha-Fagan et Schwartz (1986. p. 669), la plupart du temps «on n'essaie pas de clarifier dans quelle mesure une relation appartient en propre à la dimension individufamille et dans quelle mesure elle est tributaire du contexte environnemental dans lequel vivent les familles. " Dans certaines études, l'éventail des milieux est étroit. Par exemple, tous les enfants du Denver Youth Survey (Huizinga et al., 1991) étaient issus de secteurs à haute délinquance et socialement désorganisés. Lorsque les auteurs de recherches longitudinales ont mesuré des variables écologiques, leurs résultats n'ont pas démontré que ces variables étaient très importantes comparativement aux facteurs individuels et familiaux.

Dans l'étude Cambridge-Somerville (McCord et al., 1959), les travailleurs sociaux avaient coté l'environnement des garçons au moment de leur entrée dans le programme selon le taux de délinquance, la dégradation physique des édifices, l'existence de gangs et l'absence de bonnes organisations communautaires. Cependant, le pourcentage des garçons condamnés (jusqu'à 25 ans) n'a pas beaucoup varié selon les milieux : de $38 \%$ dans les meilleurs quartiers à $46 \%$ dans les pires. Dans des recherches subséquentes. 
McCord (1979) a trouvé que l'environnement ne contribuait pas significativement à prédire les condamnations jusqu'à 45 ans pour les crimes de violence ou contre la propriété (contrairement aux composantes de la relation éducative parents-enfants). Bien sûr, on doit se rappeler que la plupart de ces garçons avaient été sélectionnés pour le programme de prévention parce qu'on les trouvait difficiles à l'école vers 10 ans et qu'aucun d'entre eux ne provenait d'un milieu « approchant les standards de classe moyennesupérieure de certaines banlieues de Boston" (McCord et al., 1959, p. 70). Il en est résulté que la variabilité de l'environnement ne pouvait pas être très grande.

Une des recherches longitudinales prospectives qui a le plus poussé les investigations sur le milieu résidentiel est la comparaison qu'ont faite Rutter et al. (1975a, 1975b) des enfants du centre de Londres et de l'île de Wight. Ils ont considéré non pas des quartiers mais une zone urbaine et une zone rurale et ils ont étudié les troubles de comportement plutôt que la délinguance mais les résultats sont instructifs. Rutter et ses collègues ont trouvé un taux beaucoup plus élevé de troubles de comportement chez les enfants du centre de Londres et ce, indépendamment du fait que les parents y aient grandi ou s'y soient installés plus tard. Par conséquent les différences ne pouvaient être attribuées à la migration sélective des cas lourds dans les zones problèmes.

L'équipe de Rutter a démontré que, comparés à ceux de l'île de Wight. les enfants du centre de Londres souffraient davantage de discordes maritales. avaient plus souvent un seul parent, une mère ayant un trouble psychiatrique, un père criminel, un logement surpeuplé et/ou subventionné et qu'ils étaient plus susceptibles de fréquenter une école au personnel instable. Rutter (1981) en concluait que les taux de troubles de comportement étaient plus élevés au centre de Londres d'abord parce que les difficultés d'ordre familial y étaient plus fréquentes. Dans les deux milieux, à peu près le quart des enfants qui avaient un score élevé de difficultés familiales avaient de tels troubles (Rutter, 1978). Dans la mesure où la vie urbaine avait un effet sur la conduite antisociale des enfants, Rutter affirmait que c'était par l'intermédiaire de son effet sur les familles. Il n'est pas sans intérêt que Robins (1966) à St-Louis ait aussi conclu que, si on contrôlait l'antisocialité des parents, l'environnement n'aidait pas à prédire la conduite antisociale des enfants. Enfïn Robins et Hill (1966) ont montré que le pourcentage des garçons noirs qui devenaient délinquants n'avait rien à voir avec les taux de délinquance des secteurs de recensement.

Shannon $(1988,1991)$ est l'auteur d'une recherche longitudinale qui a suivi simultanément l'évolution des individus et des environnements. Tandis que son livre de 1988 n'avait pas fouillé la question de la prédiction de la délinquance individuelle à partir de facteurs individuels et environne- 
mentaux, son livre de 1991 touche à cette question. Par exemple il a montré que le type d'environnement (centre-ville versus autres) permettait de prédire la gravité des futures infractions mais pas indépendamment de l'âge, de la race et du sexe, qui sont plus importants. Il a aussi montré que les prédicteurs de la gravité des infractions variaient quelquefois selon que le lieu de résidence était le centre-ville ou un autre lieu.

On a grand besoin de recherches longitudinales plus fouillées qui suivent à la trace les modifications dans les variables individuelles, familiales et environnementales. Cependant, à la réflexion, ce type d'études soulève d'importants problèmes empiriques autant que théoriques. Nous en examinerons quelques-uns.

\section{QUELQUES PROBLÈMES EMPIRIQUES ET THÉORIQUES}

Il me semble qu'on en connaît davantage sur les influences individuelles et familiales que sur les influences environnementales. Il faudrait que les chercheurs puisent à diverses sources de données pour identifier les principales dimensions de l'environnement qui auraient, en théorie, un effet sur la délinquance. On peut les classer selon qu'elles reflètent l'environnement physique ou social, ou selon que leur influence s'exerce sur le potentiel criminel ou sur le passage à l'acte. Par exemple. les modèles antisociaux et la diffusion de valeurs déviantes peuvent influencer le potentiel criminel tandis que les occasions de crime peuvent favoriser le passage à l'acte.

L'étude des influences environnementales sur la délinquance est lacunaire dans la définition de l'environnement. La plupart des recherches se sont servi des secteurs de recensement définis par les gouvernements. Ils présentaient l'avantage de permettre l'utilisation de données publiées mais, en contrepartie. les secteurs de recensement ne pouvaient pas être les unités environnementales les plus importantes - théoriquement comme empiriquement - ni les plus influentes. Les recherches futures devraient en premier lieu identifier les principales dimensions dans lesquelles s'incarne, en théorie, l'influence du milieu sur la délinquance et, dans un deuxième temps, trouver moyen de mesurer ces dimensions fréquemment dans le temps. Les variables utilisées dans les recensements ne sont pas forcément les plus appropriées. Il serait préférable de ne pas s'en tenir aux seules caractéristiques objectives de l'environnement, mais d'aller chercher la perception individuelle de ces caractéristiques. Quelques variables écologiques ont été proposées par Tonry et al. (1991, p. 46-47) : la composition démographique, les femmes chefs de famille, la mobilité résidentielle, la pauvreté, le mode d'occupation des résidences, les organismes de loisir et la dégradation matérielle. Ils suggèrent l'utilisation de l'entrevue et des méthodes ethnographiques, de concert avec les données officielles, pour 
mesurer ces facteurs. Des mesures fréquentes sont essentielles pour suivre à la trace les changements de ces caractéristiques dans le temps.

Un autre problème est que ce qui est significatif dans l'environnement peut différer d'un individu à l'autre. Ce serait le cas pour les voisins immédiats, qui peuvent être des modèles antisociaux, des instigateurs de crimes, des complices (cf. Reiss et Farrington, 1991), ou encore qui peuvent transmettre des valeurs ou des tactiques délinquantes. Toute étude des influences environnementales devrait se pencher sur le rôle des gens du quartier. Ces derniers sont également importants en tant que victimes potentielles, vu que les délinquants s'en prennent souvent aux gens de leur entourage (cf. Wilkström, 1991, p. 213-220).

D'autres difficultés sont attribuables à la mobilité résidentielle et à l'instabilité des délinquants. Plusieurs études ont démontré que les individus condamnés déménagent plus souvent que les non-délinquants (cf. Osborn, 1980). En outre, parce qu'ils proviennent davantage de familles perturbées, les enfants antisociaux connaissent plus souvent les installations de fortune, passant quelque temps avec un parent, quelque temps avec l'autre. puis avec un grand-parent, avec une sœur ou un frère plus âgé. Dans l'étude de l'influence de l'environnement, il importe de mesurer le temps où chaque personne est exposée à de telles influences et, à partir de là, de mesurer plus précisément la population à risque (en jours-personne ou mois-personnes et pas seulement en personnes) et d'en tirer des courbes liant le temps d'exposition aux résultats.

On rencontre des problèmes semblables en regard de la famille. Le nombre de configurations familiales possibles est ahurissant, particulièrement dans le cas des enfants antisociaux (cf. Kellam et al., 1977). L'idée de suivre dans le temps les individus, leurs familles et leurs environnements est attrayante mais les familles sont en constante mutation. Pas seulement à cause de l'instabilité des relations maritales ou quasi maritales mais aussi à cause des changements inhérents au processus de croissance des enfants qui viennent à quitter le foyer pour former leur propre famille. Le nombre des familles impliquées dans une recherche longitudinale est appelé à se multiplier considérablement au cours des travaux.

Pour mesurer la délinguance, il est bien sûr essentiel de recueillir des données officielles et des données auto-révélées mais il est aussi important de cerner diverses facettes de la carrière criminelle comme la prévalence et la fréquence des infractions ainsi que le début, la durée et l'abandon de la carrière criminelle. Les facteurs environnementaux et familiaux peuvent avoir des effets divers selon les caractéristiques. C'est ainsi que la concentration d'enfants dans un quartier peut influencer l'entrée dans la carrière délinquante mais non pas la sortie, tandis que la présence d'organismes comme les églises peut n'agir que sur la sortie. Parce que l'univers social 
des gens grandit en même temps qu'eux, le rôle des parents en général peut être plus décisif sur les débuts dans la délinquance que sur le renoncement à cette forme d'activité alors que c'est l'inverse pour les facteurs environnementaux.

La recherche sur les facteurs individuels, familiaux et environnementaux de la délinquance basée rien que sur un échantillon d'individus risque de produire des résultats insatisfaisants, parce que les données écologiques sur un échantillon peuvent s'avérer insuffisantes. Ainsi, on peut se demander si l'influence écologique déterminante est autre chose que la somme des influences des individus qui composent l'entourage, indépendamment les unes des autres ou en interaction. Pour répondre adéquatement à cette question, il faut des données sur tout l'entourage plutôt que sur un échantillon seulement. Il faudrait également enquêter auprès de toute la population pour mesurer l'impact de telle ou telle proportion de familles ou d'individus antisociaux dans un milieu; par exemple, on peut penser que lorsqu'une masse critique de délinquants est atteinte dans un quartier, le sens de l'influence du voisinage change de direction : il devient criminogène alors qu'il favorisait la conformité auparavant. Crane (1991) a conclu que l'environnement avait des effets non linéaires puisque c'est seulement dans les pires quartiers des grandes villes qu'on trouve des hausses très brusques dans la prévalence des problèmes sociaux (grossesses d'adolescentes et abandon scolaire).

Il serait fort intéressant d'étudier comment des caractéristiques environnementales influencent certaines notions fondamentales des théories criminologiques classiques comme celles du fossé entre les aspirations d'une personne et ce qu'elle peut légitimement atteindre (Cloward et Ohlin, 1960), de la dévalorisation tout particulièrement ressentie par les garçons de classe inférieure qui subissent des échecs scolaires (Cohen, 1955), de l'association différentielle (Sutherland et Cressey, 1974), du lien qui unit l'individu à la société (Hirschi, 1969), de la conscience et du conditionnement par le renforcement opérant (Trasler, 1962). Il serait aussi fort utile d'étudier l'impact des caractéristiques environnementales sur les concepts clés des théories plus modernes comme la faiblesse du contrôle de soi (Gottfdredson et Hirschi, 1990) et de voir dans quelle mesure les décisions délinquantes sont influencées par des considérations futures plutôt qu'immédiates (Wilson et Herrnstein, 1985).

Bien que cet article ait pour objet central les influences environnementales et autres qui s'exercent sur la délinquance individuelle, il serait quand même intéressant d'étudier les mêmes influences, mais sur des variables écologiques comme les taux de délinquance des milieux (à partir du lieu des infractions). Plusieurs chercheurs (Baldwin et al., 1976; Reiss et Farrington, 1991 ; Wikström, 1991) ont comparé le lieu de résidence des délinquants et 
le lieu où ils commettent leurs infractions et mesuré la distance entre les deux. Il n'est pas facile de mesurer le taux d'infraction dans un quartier (pour une discussion de la question, voir Wilkström, 1991, p. 193-198). Bien que la notion de "carrière criminelle de la communauté » dépasse le cadre de cet article et en dépit des doutes émis par Laub (1987), il me semble potentiellement fructueux d'appliquer le concept de carrière criminelle à l'environnement.

Il est difficile de ne pas conclure que l'importance des facteurs environnementaux a été sous-estimée à ce jour à cause des définitions inadéquates de l'environnement et des mesures inadéquates des principales variables environnementales.

\section{CONCLUSION}

Mon article et son titre sont délibérément provocateurs. J'ai probablement passé trop de temps à discuter des recherches de Shaw et McKay (1942) et des Glueck (1950), à cause de mon agacement devant le fait qu'on a appris à des générations d'étudiants en criminologie à vénérer Shaw et McKay et à critiquer les Glueck. Je voulais mettre en évidence le fait que Shaw et McKay et les Glueck étaient également importants en criminologie, ayant apporté des contributions soignées et marquantes au savoir criminologique des années 30 et 40 , d'ailleurs encore pertinentes aujourd'bui. Quoi qu'il en soit, je voulais aussi souligner que Shaw et McKay et les auteurs de recherches écologiques qui leur ont succédé n'ont pas réussi à prouver sans ambiguïté les influences environnementales sur la délinquance, pas plus que les Glueck ni les auteurs de recherches longitudinales qui ont suivi leurs traces n'ont réussi à démontrer sans ambiguïté les influences individuelles et familiales. Les premiers n'ont pas bien mesuré les facteurs individuels et familiaux tandis que les seconds n'ont pas bien mesuré les facteurs environnementaux.

Néanmoins, il y a un consensus théorique étonnamment élevé parmi les auteurs de recherches écologiques comme Simcha-Fagan et Schwartz (1986), Gottfredson et al. (1991) et les auteurs de recherches longitudinales comme Rutter (1981), sur l'existence d'une influence écologique sur la délinquance qui s'exerce par l'intermédiaire des effets produits sur les individus et les familles. Malgré ce consensus, les preuves n'en sont pas très convaincantes. Comme on l'a dit plus haut, c'est avec de meilleures définitions et de meilleures mesures que l'importance des facteurs environnementaux émergera. Les chercheurs doivent mesurer les variables clés de chaque catégorie (et d'autres catégories comme la biologie, les pairs et l'école) et comparer systématiquement l'adéquation entre les divers modèles théoriques et les données. Il faut en étudier les effets simultanés 
comme séquentiels, réciproques comme interactifs. Les théories criminologiques doivent être élargies et inclure tous ces types de variables.

Les auteurs de travaux écologiques ont généralement mené des recherches transversales et utilisé des variables de recensement pour opérationnaliser les concepts environnementaux. L'utilisation de sources de données plus diversifiées et la réalisation de recherches longitudinales et expérimentales pourraient mener à des résultats plus concluants. Les liens de causalité seraient mieux démontrés par l'observation de changements chez les mêmes individus, les mêmes familles et les mêmes milieux à travers le temps (Farrington, 1988b). Comme Tonry et al. (1991) ainsi que Reiss (1986) l'ont dit. pour progresser, il faut procéder à des recherches longitudinales de deuxième génération, dans lesquelles on suit simultanément les individus et les environnements à partir d'une grande variété de mesures des principaux facteurs individuels, familiaux et environnementaux. Ces projets devraient être réalisés dans de grandes villes à faible émigration et aux quartiers bien définis.

De telles études permettraient de suivre les individus dans leurs déplacements d'un quartier à l'autre et, à partir de là, de prouver les influences environnementales en comparant les individus à eux-mêmes dans des analyses quasi expérimentales. En toute logique, le meilleur moyen de cerner les influences environnementales est d'étudier les mêmes personnes (ou peut-être des jumeaux identiques) dans différents milieux, tout comme le meilleur moyen d'étudier les influences individuelles et familiales est d'étudier différentes personnes dans le même environnement. De telles études pourraient également aider à voir les effets des divers types de facteurs selon l'âge et les étapes de la carrière criminelle. 11 y aurait également lieu de se pencher sur les facteurs en interaction, comme par exemple d'étudier si les changements de trajectoire individuels sont différents selon les milieux et si différents types d'individus réagissent de manière dissemblable aux mêmes influences environnementales.

De plus, il pourraît être très avantageux de faire des interventions expérimentales dans ces études longitudinales pour prouver les effets des changements de certaines caractéristiques spécifiques, individuelles. familiales ou environnementales. Ces expériences auraient des implications pratiques en indiquant des moyens de réduire la délinquance plus efficacement. Elles nous donneraient de meilleures chances de débrouiller les effets indépendants et interreliés des facteurs individuels, familiaux et environnementaux et par conséquent de développer des théories plus exhaustives de la délinquance. Ces études devraient être entreprises aussitôt que possible dans divers pays pour faire progresser le savoir sur les causes et sur la prévention de la délinquance. 


\section{Bibliographie}

BALDWIN, J. (1979), «Ecological and Areal Studies in Great Britain and the United States m. In N. Morris et M. Tonry (eds.), Crime and Justice, vol. 1 (p. 29-66), Chicago, University of Chicago Press.

BALDWIN, J., A.E. BOTTOMS et M.A. WALKER (1976), The Urban Criminal, London, Tavistock.

BERRUETA-CLEMENT, J.R., L.J. SCHWEINHART, W.S. BARNETT, A.S. EPSTEIN et D.P. WEIKART (1984), Changed Lives, Ypsilanti, Mich., $\mathrm{High} / \mathrm{Scope}$.

BOTTOMS, A.E. et P. WILES (1986), « Housing Tenure and Residential Community Crime Careers in Britain». In A.J. Reiss et M. Tonry (eds), Communities and Crime (p. 101-162), Chicago, University of Chicago Press.

BURSIK, R.J. (1986), « Delinquency Rates as Sources of Ecological Change. ». In J.M. Byme et R.J. Sampson (éds.), The Social Ecology of Crime (p. 63-74), New York, Springer-Verlag.

BURSIK, R.J. (1988), "Social disorganisation and Theories of Crime and Delinquency : Problems and Prospects », Criminology, 26, 519-551.

BURSIK, R.J. et H.G. GRASMICK (1992), «Longitudinal Neighbourhood Profiles in Delinquency : The Decomposition of Change $»$, Journal of Quantitative Crimino$\log y, 8,247-263$.

BURSIK, R.J. et J. WEBB (1982), «Community Change and Patterns of Delinquency", American Journal of Sociology, 88, 24-42.

CLARKE, R.V. (1983), «Situational Crime Prevention: Its Theoretical Basis and Practical Scope». In M. Tonry et N. Morris (éds.), Crime and Justice, vol. 4 (p. 225-256), Chicago, University of Chicago Press.

CLARKE, R.V. (1992), «Introduction». In R.V. Clarke (éd.), Situational Crime Prevention (p. 3-36), Albany, N.Y, Harrow and Heston.

CLOWARD, R.A. et L.E. OHLIN (1960), Delinquency and Opportunity, Glencoe, Ill., Free Press.

COHEN, A.K. (1955), Delinquent Boys, Glencoe, Ill., Free Press.

COHN, E.G. et D.P. FARRINGTON (1990), «Differences between British and American Criminology : An Analysis of Citations $»$, British Journal of Criminology, 30 , 467-482.

CRANE, J. (1991), "The Epidemic Theory of Ghettos and Neighbourhood Effects on Dropping Out and Teenage Childbearing ", American Journal of Sociology, 96, 1226-1259.

ELLIOT, D.S., D. HUIZINGA et S. AGETON (1985), Explaining Delinquency and Drug Use, Beverly Hills, Calif,, Sage.

ELLIOT, D.S., D. HUIZINGA et S. MÉNARD (1989), Multiple Problem Youth, New York, Springer-Vertag.

FARRINGTON, D.P. (1979), «Longitudinal Research on Crime and Delinquency». In N. Morriss et M. Tonry (éds.), Crime and Justice, vol. 1 (p. 289-348), Chicago, University of Chicago Press. 
FARRINGTON, D.P. (1988a), «Advancing Knowledge about Delinquency and Crime : The need for a Coordinated Programme of Longitudinal Research », Behavioural Sciences and the Law, 6, 307-331.

FARRINGTON, D.P. (1988b), « Studying Changes within Individuals : The Causes of Offending ». In M. Rutter (ed.) Studies of Psycho-social Risk (p. 158-183), Cambridge, Cambridge University Press.

FARRINGTON, D.P. (1992a), «Criminal Career Research in the United Kingdom ", British Journal of Criminology, 32, 521-536.

FARRINGTON, D.P. (1992b), «Explaining the Beginning, Process and Ending of Antisocial Behaviour from Birth to Adulthood\%. In J. McCord (éd.), Facts, Frameworks and Forecasts (p. 253-286), New Brunswick, N..J., Transaction.

FARRINGTON, D.P. (1992c), «Juvenile Delinquency ». In J.C. Coleman (éd.), The school Years, $2^{\mathrm{e}}$ éd. (p. 123-163), London, Routledge.

FARRINGTON, D.P. (1993), «Motivations for Conduct Disorder and Delinquency », Development and Psychopathology, 5, p. 225-241.

FIREBAUGH, G. (1978), «A rule for Inferring Individual-Level Relationships from Aggregate Dala m, American Sociological Review, 43, 557-572.

GLASER, D. et K. RICE (1959), “Crime, Age, and Unemployment ", American Sociological Review, 24, p. 679-686.

GLUECK, S. et E.T. GLUECK (1930), Five Hundred Criminal Careers, New York, Knopf.

GLUECK, S. et E.T. GLUECK (1934), One Thousand Juvenile Delinquents, Cambridge, Mass., Harvard University Press.

GLUECK, S. et E.T. GLUECK (1937), Later Criminal Careers, New York, Commonwealth Fund.

GLUECK, S. et E.T. GLUECK (1940), Juvenile Delinquents Grown Up, New York, Commonwealth Fund.

GLUECK, S. et E.T. GLUECK (1943), Criminal Careers in Retrospect, New York, Commonwealth Fund

GLUECK, S. et E.T. GLUECK (1950), Unravelling Juvenile Delinquency, Cambridge, Mass., Harvard University Press.

GLUECK, S. et E.T. GLUECK (1968), Delinguents and Non-Delinquents in Perspective, Cambridge, Mass., Harvard University Press.

GOLD, M. (1987), «Social Ecology». In H.C. Quay (éd.), Handbook of Juvenile Delinquency, (p. 62-105), New York, Wiley.

GOTTFREDSON, D.C., R.J. McNEIL et G.D. GOTTFREDSON (1991), « Social Area Influences on Delinquency: A Multi-Level Analysis ». Journal of Research on Crime and Delinquency, 28, 197-226.

GOTTFREDSON, M.R. et T. HIRSCHI (1987), «The Methodological Adequacy of Longitudinal Research on Crime ", Criminology, 25, 581-614.

GOTTFREDSON, M.R. et T. HIRSCHI (1990), A General Theory of Crime, Stanford, Calif, Stanford University Press.

GOTTFREDSON, S.D., R.B. TAYLOR (1986), « Person-Environment Interactions in the Production of Recidivism». In J.M. Byme et R.J. Sampson (eds.), The Social Ecology of Crime (p. 133-155), New York, Springer-Verlag. 
HAMPARIAN, D.M., R. SCHUSTER, S. DIMITZ et J.P. CONRAD (1978), The Violent Few, Lexington, Mass., Heath.

HAWKINS, J.D., E. VON CLEVE et R.F. CATALANO (1991), «Reducing Early Childhood Aggression : Results of a Primary Prevention Programme", Journal of the American Academy of Child and Adolescent Psychiatry, 30 208-217.

HIRSCHI, T. (1969), Causes of Delinquency, Bcrkeley, Calif., University of California Press.

HIRSCHI, T. et H.C. SELVIN (1967), Delinquency Research, New York, Free Press.

HOPE, T. et J. FOSTER (1992), "Conflicting Forces : Changing the Dynamics of Crime and Community on a "Problem" Estate ", British Journal of Criminology, $32,488-504$.

HOPE, T. et M. SHAW (eds) (1988), Communities and Crime Reduction, London, Her Majesty's Stationery Office.

HUZINGA, D., F-A. ESBENSEN et A.W. WEIHER (1991), «Are There Multiple Paths to Delinquency? , Journal of Criminal Law and Criminology, 82, 83-118.

JONASSEN, C.T. (1949), "A Re-Evaluation and Critique of the Logic and some Methods of Shaw and McKay », American Sociological keview, 14, 608-614.

JOHNSTONE, J.W.C. (1978), «Juvenile Delinquency and the Family: A Contextual Intecpretation", Youth and Society, 9, 299-313.

KELLAM, S.G., M.E. ENSMINGER et R.J. TURNER (1977), « Family Structure and the Mental Health of Children ", Archives of General Psychiatry, 34, 1012-1022.

KELlLING, G.L., T. PATE, D. DIECKMAN et C.E. BROWN (1976), "The Kansas City Preventive Patrol Experiment: A Summary Report». In G.V. Glass (éd.), Evaluation Studies Review Annual, vol. 1, Beverly Hills, Calif., Sage.

LAUB, J.H. (1987), « Rediscovering the Importance of Cities, Neighbourhoods and Crime », Journal of Quantitative Criminology, 3, 83-93.

LAUB, J.H. et R.J. SAMPSON (1988), «Unravelling Families and Delinquency : a Reanalysis of the Gluecks' Data », Criminology, 26, 355-380.

LAUB, J.H. et R.J. SAMPSON (1991), "The Sutherland-Glueck Debate: On the Sociology of Criminological Knowledge », American Journal of Sociology, 96, 1402-1440.

McCORD, J. (1979), "Some Child-Rearing Antecedents of Criminal Behaviour in Adult Men., Journal of Personality and Social Psychology, 37, 1477-1486.

MCCORD, W., J. McCORD et I.K. ZOLA (1959), Origins of Crime, New York, Colombia University Press.

MORASH, M. et L. RUCKER (1989), «An Exploratory Study of the Connection of Mother's Age at Childbearing to her Children's Delinquency in Four Data Sets *, Crime and Delinquency, 35, 45-93.

OSBORN, S.G. (1980), «Moving Home, Leaving London and Delinquent Trends", British Journal of Criminology, 20, 54-61.

REISS, A.J. (1951a), "Delinquency as the Failure of Personal and Social Controls ", American Sociological Review, 16, 96-207.

REISS, A.J. (1951b), «Unravelling Juvenile Delinquency. II. An Appraisal of the Research Methods ", American Journal of Sociology, 57, 115-120. 
REISS, A.J. (1986), * Why Are Communities Important in Understanding Crime ?». In A.J. Reiss et M. Tonry (éds.), Communities and Crime (p. 1-33), Chicago, University of Chicago Press.

REISS, A.J. et D.P. FARRINGTON (1991), «Advancing Knowledge About CoOffending : Results from a Prospective Longitudinal Survey of London Males $*$, Journal of Criminat Law and Criminology, 82, 60-395.

ROBINS, L.N. (1966), Deviant Children Grown Up, Baltimore, Williams and Wilkins.

ROBINS, L.N. et S.Y. HILL (1966), «Assessing the Contributions of Family Structure, Class and Peer Groups to Juvenile Delinquency», Journal of Criminal Law. Criminology and Police Sciences, 57, 325-334.

ROBINSON, W.S. (1950), «Ecological Correlations and the Behaviour of Individuals », American Sociological Review, 15, 351-357.

ROSENBAUM, J.E. et S.J. POPKIN (1991), «Employment and Earnings of Low Income Blacks Who Move to Middle-Class Suburbs». In C. Jencks et P.E. Peterson (eds.), The Urban Underclass (p. 342-356), Washington, D.C., The Brooking Institution.

ROSS, R.R. et B.D. ROSS (1988), «Delinquency Prevention Through Cognitive Training ", New Education, 10, 70-75.

RUTTER, M. (1978), «Family, Area and School Influences in the Genesis of Conduct Disorders $»$ In L.A. Hersov, M. Berger et D. Shaffer (éds.), Aggression and Antisocial Behaviour in Childhood and Adolescence (p. 95-113), Oxford, Pergamon.

RUTTER, M. (1981), «The City and the Child », American Journal of Orthopsychiatry, 51, 610-625.

RUTTER, M., A. COX, C. TUPLING, M. BERGER et W. YULE (1975a), «Attainment and Adjustment in Two Geographical Areas: I. The Prevalence of Psychiatric Disorder , British Journal of Psychiatry, 126, 493-509.

RUTTER, M., B. YULE, D. QUINTON, O. ROWLANDS, W. YULE et M. BERGER (1975b), "Attainment and Adjustment in Two Geographical Areas : III. Some Factors Accounting for Area Differences \#, British Journal of Psychiairy, 126, 520-533.

SAMPSON, R.J. et W.B. GROVES (1989), «Community Structure and Crime : Testing Social-Disorganisation Theory , American Journal of Sociology, 94, 774-802.

SAMPSON, R.J. et J. LAURITSEN (1990), « Deviant Lifestyles, Proximity to Crime, and the Offender-Victim Link in Personal Violence", Journal of Research in Crime and Delinquency, 27, 110-139.

SHERMAN, L.W., P.R. GARTIN et M.E. BUERGER (1989), «Hot Spots of Predatory Crime: Routine Activities and the Criminology of Place», Criminology, 27, 27-55.

SCHLOSSMAN, S. et M. SEDLAK (1983), «The Chicago Area Project Revisited», Crime and Delinquency, 29, 398-462.

SCHMID, C.F. (1960), «Urban Crime Areas : Part II. », American Sociological Review, 25, 655-678.

SCHUERMAN, L. et S. KOBRIN (1986), "Community Careers in Crime». In A.J. Reiss et M. Tonry (éds.), Communities and Crime (p. 67-100), Chicago, University of Chicago Press. 
SHANNON, L.W. (1988), Criminal Career Continuity, New York, Human Sciences Press.

ShanNON, L.W. (1991), Changing Patterns of Delinquency and Crime, Boulder, Colo., Westview.

SHAW, C.R. et H.D. McKAY (1942), Juvenile Delinquency and Urban Areas, Chicago, University of Chicago Press.

SHAW, C.R. et H.D. McKAY (1949), "Rejoinder ", American Sociological Review, $14,614-617$.

SHAW, C.R. et H.D. McKAY (1969), Juvenile Delinquency and Urban Areas (rev. ed.), Chicago, University of Chicago Press.

SHAW, C.R., F. ZORBAUGH, H.D. MCKAY et L.S. COTTRELL (1929), Delinquency Areas, Chicago, University of Chicago Press.

SHORT, J.F. (1969), "Introduction to the revised edition". In C.R. Shaw et H.D. McKay (1969), Juvenile Delinquency and Urban Areas (xxv-liv), Chicago, University of Chicago Press.

SIMCHA-FAGAN, O. et J.E. SCHWARTZ (1986), « Neighbourhood and Delinquency : An Assessment of Contextual Effects , Criminology, 24, 667-703.

SUTHERLAND, E.H. (1939), Principles of Criminology ( $3^{e} \mathrm{ed}$.), Chicago, Lippincott.

SUTHERLAND, E.H. et D.R. CRESSEY (1974), Criminology, $9^{e} e ́ d .$, Philadelphia, Lippincott.

TONRY, M., L.E. OHLIN et D.P. FARRINGTON (1991), Human Development and Criminal Behaviour (with contributions by K. Adams, F. Earls, D.C. Rowe, R.J. Sampson, R.E. Tremblay), New York, Springer-Verlag.

TRASLER, G.B. (1962), The Explanation of Criminality, London, Routledge and Kegan Paul.

TREMBLAY, R.E., J. McCORD, H. BOILEAU, P. CHARLEBOIS, C. GAGNON, M. LEBLANC et S. LARIVEE (1991), * Can Disruptive Boys be Helped to Become Competent ? ", Psychiatry, 54, 148-161.

WALLIS, C.P. et R. MALIPHANT (1967), «Delinquent Areas in the county of London : Ecological Factors », British Journal of Criminology, 7, 250-284.

WEST, D.J. (1982), Delinquency: Its Roots, Careers and Prospects, London, Heinemann.

WEST, D.J. et D.P. FARRINGTON (1973), Who Becomes Delinquent?, London, Heinemann.

WEST, D.J. et D.P. FARRINGTON (1977), The Delinquent Way of Life, London, Heinemann.

WIKSTROM, P-O.H. (1985), Everyday Violence in contemporary Sweden, Stockholm, National Council for Crime Prevention.

WIKSTROM, P-O.H. (1991), Urban Crime, Criminals and Victims, New York, Springer-Veriag.

WILSON, J.Q. et R.J. HERRNSTEIN (1985), Crime and Human Nature, New York, Simon and Schuster.

WOLFGANG, M.E., R.M. FIGLIO et T. SELLIN (1972), Delinquency in a Birth Cohort, Chicago, University of Chicago Press.

WOLFGANG, M.E., T.P. THORNBERRY et R.M. FIGLIO (1987), From Boy to Man, From Delinquency to Crime, Chicago, University of Chicago Press. 